\title{
Reduction by a Nanotechnology Device of Serum Lactate Levels and Heart Rate in Athletes Undergoing to Resistance Exercises
}

\author{
Johrian Ara ${ }^{1}$, De Martino Angelo ${ }^{2}$, Beninati Simone ${ }^{2}$, Pumpo Alessandro 3 \\ ${ }^{1}$ Department of Traditional Medicine, University of Yerevan, Yerevan, Armenia \\ ${ }^{2}$ Department of Biology, University of Tor Vergata, Rome, Italy \\ ${ }^{3}$ Department of Clinical Biochemistry, University San Raffaele, Rome, Italy
}

Email address:

beninati@bio.uniroma2.it(B. Simone)

\section{To cite this article:}

Johrian Ara, De Martino Angelo, Beninati Simone, Pumpo Alessandro. Reduction by a Nanotechnology Device of Serum Lactate Levels and Heart Rate in Athletes Undergoing to Resistance Exercises. Journal of Biomaterials. Vol. 2, No. 1, 2018, pp. 16-19.

doi: $10.11648 /$ j.jb.20180201.14

Received: April 28, 2018; Accepted: May 24, 2018; Published: July 4, 2018

\begin{abstract}
The aim of the present study was to demonstrate the effect of the T-NES ${ }^{\circ}$-HiT Tech patch system, on serum lactate levels and on the heart rate, of 60 triathletes, subjected to a resistance test. The active central portion of the patch contains a nanotechnological material (silver micronized with plasma vaporization) that is appropriately activated with a technology device, which uses optical radiation, magnetic fields, electric fields and acoustic waves specially sequenced. The patch transfers an Extremely Low Frequency (ELF) magneto-electric pulse by ion exchange. The ELF signal emitted by the device, increases the electromagnetic coherence of the systemic cellular electrical signals, which shows a better management of the muscular contraction, both bilaterally and between the agonist and antagonist districts and also a better management of oxygen and lactate, elements that lead to increased proprioception. The final result is manifested in the activation of the transmembrane ion channels, thus providing sufficient energy to the phosphate groups of the ADP, avoiding the deprotonation of excess lactic acid, present in the blood stream and therefore qualitatively improving the work of muscle contraction and aerobic capacity. Following these effects the T-NES ${ }^{\circledR}$-HiT Tech patch system has led to a significant decrease in heart rate and serum lactate production in the athletes examined.
\end{abstract}

Keywords: Endurance, Micronized Silver Patch Device, Serum Lactate, Heart Rate

\section{Introduction}

The induction of a cellular effect, by a nano- technological device has as its premise the ability to guide the various metabolic phases through mechanisms that act on all interfering phenomena and through the induction of repairing chemical cascades in sequence. Evolutionary morphogenesis is a 4-dimensional creative process of which a temporal one: a $\Delta \mathrm{qV}$ of volume in a $\Delta \mathrm{qT}$ of four-dimensional time, guided by the membrane sensors, the cytoskeleton and the morphogen system. The realization of the biological project is the result of the actions on the molecular machines, of the internal and external chemical-physical information of the system, integrated, processed and translated by the genome and then realized through gene expression. The attractor, to which the buffer system is addressed, can be guided through simple or complex physical signals, containing information that accelerates the kinetics of the molecular cascades.

Any form of energy impacts a biological system by modifying the $\Delta \mathrm{G}^{\circ}$ and the functional dynamics of some multi-potential signaling devices such as $\mathrm{Ca}^{2+}$, or $\mathrm{G}$ protein coupled receptors, but also the gene transcription system, which is able to modify the activation or deactivation of molecular switches. It is possible, to influence cellular functionality from the outside with appropriate physical signals [1], inducing functional changes by piloting some important biochemical reactions [2], improving the resistance of cells to hypoxia [3]. Electromagnetic fields (EMF) are able to modify chemical reactions in polar aqueous solutions, as shown by the Piccardi effect.

Complex EMFs are also able to modify the ionic and protonic flow of cell membranes, modifying the degree of 
polarization and permeability. EMFs modify cellular signaling by accelerating electronic transfer, through direct action on gap junctions and signal molecules, including morphogens [1]. This is possible using complex electric-acoustic-optical magnetic field instruments, different from simple fields for geometry, created through the production of harmonics by each portion of the wave. By sequencing a series, albeit limited, of combinations of frequency, time, field geometry and signal intensity, an electrical/ informational signal can be transferred to the reference target. The information must be sufficiently complex to activate simultaneously all the chemical cascades dedicated to the process to be obtained, starting from the formation of the fibrillary networks [4], passing through the ionic and protonic channels [5], continuing with the cellular signaling short range and long range. The fundamental characteristic of information is the possibility of being codified in a virtually infinite number of ways. The use of specific devices unites all the ways. The amount of information that can be carried by a radio signal is proportional to its frequency (modulation theorem). By modulation, we mean the transmission technique of an electric or electromagnetic signal called modulating (possibly representing an information), by means of another electric or electromagnetic signal called carrier, which has the purpose of converting the signal from base band to translated band according to the modulation theorem. The signals to be modulated can represent the most diverse information: i.e. audio, video, data.

Endogenous magnetic fields are fluctuating ultra-dry fields, derived from changes in the electric field given by variable dipoles, intra and extra cellular, in reaction to the dynamics of ionic flow and their interaction with polar proteins. They are continuously variable and can be analyzed with non-linear mathematical systems. Their non-linearity is closely linked to the non-linearity of the ionic flow. Given their intensity, in the picoTesla order, they can be influenced by external devices, with which they tend to create a balance [6]. The endogenous fields are responsible, in part, together with the free charges of proteins and the states of activation of the water molecules [4], of the electromagnetic buoyancy of the cytosol, i.e. the ability to fluctuate the charged micro particles. This is also a physical characteristic of the cytosol which determines its relative density. Since endogenous fields are so thin, that they can be strongly conditioned, this helps us to better understand how, an infinitesimal amount of energy (i.e. the patch T-NES ${ }^{\circledR}-\mathrm{HiT}$ device) is enough to adapt the cellular work to the conditions present moment by moment [7].

Anaerobic power and anaerobic capacity are essential elements in many sports. Anaerobic performance is one of the main characteristics of brief and explosive exercise in different sports. Anaerobic power is the ability to generate energy through the phosphagen system in brief and intensive exercises and anaerobic capacity is the ability to combine the energies from glycolysis and the phosphagen system [8]. During intense exercise, as in sprinting, phosphates are the fastest and most available source of ATP. The main metabolic pathway for the regeneration of ATP during cardiovascular and endurance exercise is almost exclusively mitochondrial respiration (aerobic system), which initially shares the same metabolic pathway of the anaerobic process of glycolysis. It is wrong to believe that the body's energy systems work independently. In fact, the three energy systems, work together cooperatively to produce ATP. Through glycolysis, blood glucose and muscle glycogen are converted into pyruvate, which, depending on the intensity of the exercise, will enter the mitochondria or be converted into lactate. At levels of exercise intensity below the anaerobic threshold, pyruvate enters the mitochondria and muscle contraction continues through aerobic ATP production. While at intensity levels above the anaerobic threshold the ability to produce ATP through mitochondrial respiration is impaired, and pyruvate is converted to lactate. Lactate therefore results as a by-product of the metabolism that is produced during the catabolism or the energetic use of carbohydrates. The metabolic pathways that support the training intensity above the "anaerobic threshold" are able to sustain muscle contraction only for short periods, thus limiting performance. It is at this point that high-intensity physical exercise is compromised, because the energy systems of glycolysis and phosphagen that are sustaining muscle contraction continued above the anaerobic threshold can produce ATP at a high rate, but are able to do it only for a limited period [9]. Thus, energy for physical activities requires a mixture of all energy systems. The lactic acid begins to accumulate in the muscles and in the blood when the speed of synthesis exceeds the speed of disposal. Approximately, this condition triggers when during an intense exercise the heart rate exceeds $80 \%$ (for the untrained) and $90 \%$ (for the most trained) of the maximum heart rate.

The T-NES ${ }$-HIT Tech., Micronized silver patch device, acts as a complex magneto-electric carrier. Its action manifests itself, inducing a direct activation of protonic and ionic flows from the extracellular matrix, passing through glycocalyx, to the erythrocyte intracellular environment. This characteristic leads to the activation of the transmembrane ion channels, thus providing sufficient energy to the phosphate groups of the ADP, not allowing the acid deprotonation excess lactic acid in the blood stream, qualitatively improving the work of muscle contraction [10].

\section{Materials and Methods}

\subsection{Experimental Procedures}

The study was conducted on 60 male athletes (triathlon competitors) with an average age of $27.63 \pm 1.54$ years, an average weight of $77.23 \pm 1.90 \mathrm{~kg}$ and a BMI factor of $23.73 \pm$ 0,91 . The subjects played, after 3 min. of initial warm up, 6 total trials, seven days apart from each other, of 10, 20, 30, 40 and $50 \mathrm{~min}$. running at $9 \mathrm{~km} / \mathrm{h}$ with an incremental gradient of $0.5 \%$ every 10 min., using a Technogym ${ }^{\circledR}$ Myrun treadmill (Technogym ${ }^{\circledR}$ SpA Via Calcinaro 286147521 Cesena, FC). The heart rate expressed in beats/ min, has been determined by means of a Polar M460 HR Heart Rate Monitor (Polar Italy), while the serum lactate level, expressed in $\mathrm{mmol} / \mathrm{L}$, was evaluated using the LACTATE PRO 2 device (Arkray тм Global Business.inc, Kyoto, Japan). The maximum heart rate 
(HRmax) is the highest heart rate an individual can achieve without severe problems through exercise stress, and generally decreases with age. Since HRmax varies by individual, the most accurate way of measuring HRmax is via a cardiac stress test. In this test, the subjects underwent to a controlled physiologic stress while being monitored.

The device, T-NES®- HIT Tech., (New Era Sport Technologies, Italy) is a disposable patch $(5 \mathrm{~cm} \mathrm{x} 7 \mathrm{~cm})$, with an active central pad, based on micronized silver. The pad contains a nanotechnological material (silver micronized by plasma vaporization) that is appropriately treated with optical, magnetic, electrical and acoustic signals, in order to transfer very thin extremely low frequency (ELF) magnetoelectric signals in the order of nanoTesla, such as to induce an action on cellular glycocalics, fundamental for cellular communication and recognition, by a direct activation of protonic and ionic fluxes and to guarantee a better transport of $\mathrm{O}_{2}$ in the blood cells. These signals also optimize the binding consistency in the musculoskeletal cells and lower the impedance in the nerve cells. This results in an increase in "endurance". The measurements were conducted on the same 60 athletes in two total tests. The first test without the application of the T-NES ${ }^{\circ}$ - HIT Tech, patch (control) and the second test with the application of the patch.

\subsection{Statistical Analyses}

The statistical analysis was performed using the Mann-Whitney $U$ test for intergroup comparisons. Spearman's rank correlation test and the linear regression analysis were performed to analyze correlations between groups and the duration of tests. Values were expressed as the mean \pm SD. $P<0.05$ was regarded as statistically significant

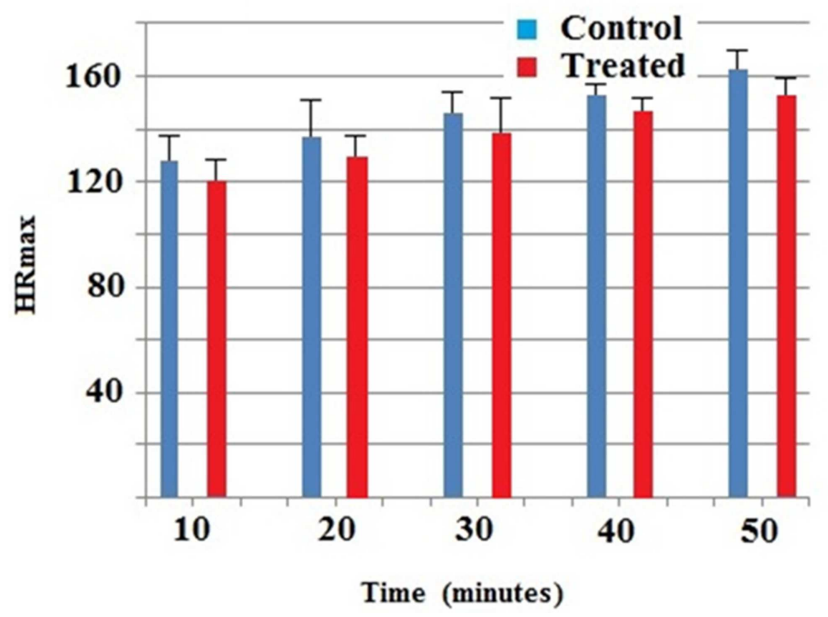

Figure 1. HRmax variation expressed as heart beats per minute (bpm) during resistance tests in treated-athletes compared to control, as described in the Materials and Methods. The data represent the average of at least 60 observations for each time $\pm S D$. Statistically significant $(P<0.05)$.

\section{Results and Discussion}

Moderate electric fields (MEF) have shown interesting influences on the permeability and diffusion across membranes of eukaryotic [11] and prokaryotic cells [12]. Electroporation is a membrane phenomenon which has been known for some time. By applying an external electric field to a cell, physically modeled as a conductive body surrounded by a dielectric layer, electric charges build up on the cell membrane and consequently pore formation occurs [13]. Most publications on electroporation focus on the effect of high intensity pulsed electric fields (PEF), but mild fields, such as those under electromagnetic field (MEF) (typically 1-1,000 $\mathrm{V} / \mathrm{cm}$ ) have received little attention. Several biological processes, such as limb regeneration in amphibia, fracture repair in rabbits and humans, liver regeneration in rats, are affected by non-thermal low frequency MEF [14]. At the cellular level, MEFs appear to be able to modify transcription and differentiation, synthesis of specialized proteins, response to hormones and release of neurotransmitters, growth and the DNA synthesis of some cells, such as chick chondrocytes or murine embryonal carcinoma cell line F9 [15]. MEFs are also able to enhance in vitro the growth rate of endothelial cells and angiogenesis [16] and the tumoricidal activity of nonparenchymal liver cells $[17,18]$

The execution of a physical exercise involves the functional integration of the respiratory, cardiovascular and muscular systems, in order to cope with the increased energetic demand and the increase of respiratory gases $\left(\mathrm{O}_{2}\right.$ and $\left.\mathrm{CO}_{2}\right)$ in the skeletal musculature. The respiratory and the cardiovascular apparatus have a wide functional reserve and their possible alteration is clinically manifested, only when this is greatly reduced. In fact, many of the methods suitable for assessing the function of these rest units, do not adequately reflect their reserve capacity. The reduction of this functional reserve, however, contributes significantly to the reduction of the exercise tolerance. The magnetic fields used for medical purposes have shown that the interactions between the MEF and human cells lead to changes in cellular metabolism. The individual components of a biological system, immersed in a $\mathrm{MEF}$, undergo a stress due to the electrical charges present on molecules, ions and free radicals.

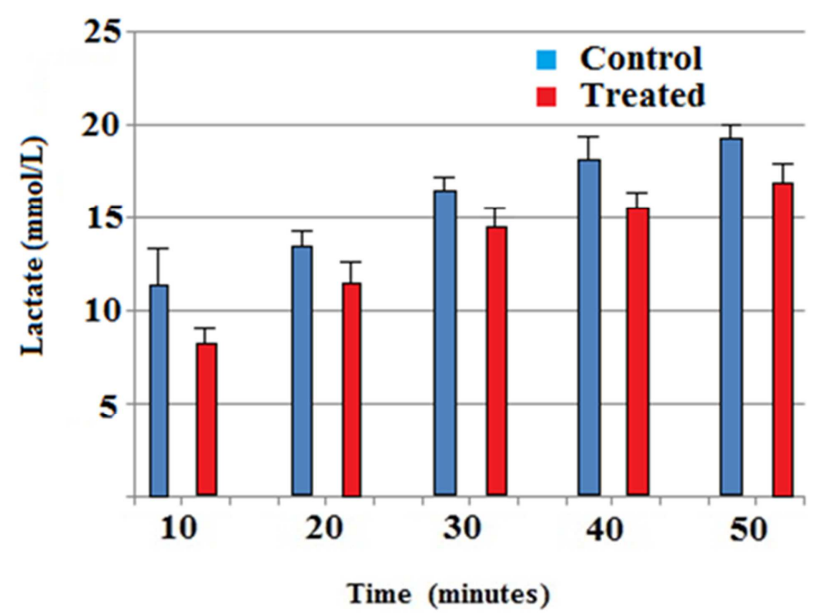

Figure 2. Variation of serum lactate levels during resistance tests in treated-athletes compared to control, as described in the Materials and Methods. The data represent the average of at least 60 observations for each time $\pm S D$. Statistically significant $(P<0.05)$. 
The objective of this investigation was to verify if the ELF fields, generated by the nanotechnology device T-NES ${ }^{\circledR}$ - HIT Tech., influence the stimulation of the human muscular apparatus, evaluating the potential reduction of serum lactate levels and the heart rate of 60 triathletes, subjected to a resistance test (see Materials and Methods). The effect of the application of T-NES ${ }^{\circledR}-H I T$ Tech. micronized silver patch device to triathletes is shown as HRmax (Figure 1) and serum lactate levels (Figure 2).

The values of the decreases obtained by the T-NES $®$ - HIT Tech. patch system, during the five exercises, for serum lactate, were $7 \%\left(10^{\prime}\right), 6.16 \%\left(20^{\prime}\right), 6.00 \%$ (30'), 4.25\% (40') and $7 \%$ (50'). Only the exercise for 40 ' (4.25\%), has deviated from the $6.5 \%$ reduction average obtained for the other exercise times. The values of the decreases during the five exercises for the heart rate were $27.63 \%\left(10^{\prime}\right), 13.50 \%$ $\left(20^{\prime}\right), 11.80 \%(30 '), 13.74 \%(40 ')$ and $12.65 \%(50 ')$. Only the exercise for $10^{\prime}(27.63 \%)$ has deviated from the $13 \%$ reduction average, obtained for the other exercise times. In conclusion, the average reduction gained in both the parameters observed, thanks to T-NES $\AA$ - HIT Tech. patch system, was of about $6.5 \%$ for the HRmax and $13 \%$ for serum lactate levels compared to control.

\section{Conclusion}

The ELF magnetic field of a T-NES $₫$-HIT Tech micronized silver patch device, applied to triathletes, subjected to a resistance test, showed a statistically significant reduction in both heart rate and serum lactate levels in all the exercises performed. In order to make the most of their skills, in the field of competitive sports, there are often many difficulties, related to the response of one's body to fatigue. Overcoming this obstacle, thus being able to express all possible performance, is connected to that training path that sees the athlete forced to improve skills with constant exercise. Based on the results presented, the T-NES ${ }^{\circledR}$-HIT Tech function, is to provide an aid by maintaining two basic characteristics at physiological state, heart rate and serum lactate levels, thus offering an additional element to improve sports performance.

\section{Acknowledgements}

We are grateful to Beninati Simone and De Martino Angelo from University of Tor Vergata, Roma, Italy, for preparation and critical reading of the manuscript.

\section{References}

[1] F. Crescentini, "Complex Magnetic Fields as broadcasting of bioactive information”. MP\&BN Editor, 2013.

[2] F. Crescentini, Neo-osteomorphogenesis guided with CMF. SIMPLE Editor. 2009.
[3] F. Crescentini, The epistemological bases of the biological information system. SIMPLE Editor. 2011.

[4] B. Cuccurazzu, L. Leone, MV. Podda, R. Piacentini, E. Riccardi, C. Ripoli, G. B. Azzena, C. Grassi. Exposure to extremely low-frequency $(50 \mathrm{~Hz})$ electromagnetic fields enhances adult hippocampal neurogenesis in C57BL/6 mice. Exp. Neurol. 2010; 226(1):173-182.

[5] L. Montagnier, J. Aissa, E. Del Giudice, C. Lavallee, A. Tedeschi, G. Vitiello. DNA waves and water. Journal of Physics: Conference Series 2011; 306.

[6] Y. Zimmels. Thermodynamics in the presence of electromagnetic fields. Phys Rev E Stat Phys Plasmas Fluids Relat Interdiscip Topics. 1995; 52(2):1452-1464.

[7] S. J. Williamson, L. Kaufman. Evolution of neuromagnetic topographic mapping. Brain Topogr. 1990; 3(1): 113-127.

[8] S. A. Plowman, D. L. Smith. Anaerobic Metabolism during Exercise. Sports-Specific Rehabilitation, 2007; 23:213-230.

[9] D. R. Bassett Jr, E. T. Howley. Limiting factors for maximum oxygen uptake and determinants of endurance performance. Med. Sci. Sports Exerc. 2000; 32(1):70-84.

[10] S. Altobello, A. Filippone, L. Grillo, A. De Martino, S. Beninati, A. Pumpo. Improved Endurance Gain in BALB/ C Mice by a Nanotechnology Medical Device. International Journal of Biomedical Materials Research. 2018; 6(1): 13-19.

[11] P. J. R. Schreier, D. G. Reid, P. J. Fryer. Enhanced diffusion during the electrical heating of foods. Int. J. Food Sci. Technol. 1993; 28: 249-260.

[12] L. Loghavi, S. K. Sastry, A. E. Yousef. Effect of moderate electric field frequency on growth kinetics and metabolic activity of Lactobacillus acidophilus. Biotechnol Prog. 2008; 24: $148-153$.

[13] J. Deng, K. H. Schoenbach, E. S. Buescher, P. S. Hair, P. M. Fox, S. J. Beebe SJ. The effect of intense submicrosecond electrical pulses on cells. Biophys J. 2003; 84: 2709-2714.

[14] C. A. Bassett, R. J. Pawluk, A. A. Pilla. Augmentation of bone repair by inductively coupled electromagnetic fields. Science. 1974; 184 (4136):575-7.

[15] T. Akamine, H. Muramatsu, H. Hamada, T. Sakou. Effects of pulsed electromagnetic field on growth and differentiation of embryonal carcinoma cells. J. Cell. Physiol. 1985; 124(2):247-254.

[16] G. P. Yen-Patton, W. F. Patton, D. M. Beer, B. S. Jacobson. Endothelial cell response to pulsed electromagnetic fields: stimulation of growth rate and angiogenesis in vitro. J Cell Physiol. 1988; 134(1):37-46.

[17] M. Malter, G. Schriever, R. Kühnlein, R. Süss. Tumoricidal cells increased by pulsating magnetic field. Anticancer Res. 1987 May-Jun; 7(3 Pt B):391-3

[18] W. R. Adey, J. C. Lin. Biological effects of radio frequency electromagnetic radiation. Electromagnetic Interaction with Biological Systems. Plenum 1999; 109-140. 